\title{
Study on Bubble Velocity under Bubble Flow Scenario in the Horizontal Water Transmission Pipeline
}

\author{
Zhu Yan ${ }^{1,}$ a , Wu Chenguang ${ }^{2, b}$ and Yuan Yixing ${ }^{3, c}$ \\ ${ }^{1}$ School of Municipal and Environmental Engineering, Harbin Institute of Technology, 73 Huanghe \\ Road, Harbin, China. \\ ${ }^{2}$ School of Municipal and Environmental Engineering, Harbin Institute of Technology, 73 Huanghe \\ Road, Harbin, China. \\ ${ }^{3}$ School of Municipal and Environmental Engineering, Harbin Institute of Technology, 73 Huanghe \\ Road, Harbin, China. \\ aifengtian@126.com, bwu.c.g@126.com, cyyx1957@163.com
}

Keywords: bubble velocity; bubble motion model; continuous photographing; mean bubble diameter; sliding friction coefficient

Abstract. In order to put forward some feasible suggestions for the air emission in water transmission pipeline, this article mainly studies the bubble velocity under bubble flow scenario in the horizontal water transmission pipe. It was assumed that the bubble in the horizontal pipe could be regarded as a rigid body with a spherical shape, and the concept of sliding friction was introduced into the stress analysis to represent for the interaction between the bubble and the pipe wall. Taking all the forces on the bubble into consideration, a completely theoretical model was established to calculate the bubble velocity. During the experiments, the method of continuous photographing was employed to record the bubble size and velocity, and the experimental data was used to verify the accuracy of the model. It was found that the experimental data fitted well with the theoretical model after multiplying a correction factor. Ultimately, the corrected model was used to study the effects of pipe diameter, pipe variety, pipe pressure and temperature on the bubble size and bubble velocity, and it was proposed that more air release valves should be installed at the terminal part of the water transmission pipeline for air emission.

\section{Introduction}

Gas-liquid two-phase flow is a normal state in the water transmission pipeline, and the presence of air will not only increase energy consumption for water supply, but also lead to pipe burst at some locations during the hydraulic transition process when the air volume reaches a certain extent. In order to provide some feasible suggestions for the air discharging, it is necessary to know the characteristics of bubble motion in the water transmission pipeline. Consequently, study on bubble velocity in the horizontal pipe is conducive to understand the characteristics of bubble motion.

Many other industries, e.g. the petroleum industry, have conducted a lot of research on bubble velocity in the pipes with different diameters and different angles. The slip velocity between bubble and water could be calculated using a theoretical method, which needs to know the average void fraction at the pipe cross section by using optical fiber probe technique [1] or the electrical resistance tomography technique [2] and so on. In theory, it is a reliable method to obtain the bubble velocity at a certain position, but it is difficult to apply these techniques into real world application, because the measurement of the average void fraction may be inaccurate due to the asymmetrical distribution of bubbles at the pipe cross section. Moreover, the high-speed photography technology [3] as well as the particle image velocimetry technology [4] has been widely utilized to detect the bubble velocity, which needs the pipes to be transparent and has stringent requirements on the photographing light source. Thus, all the technologies mentioned above have some limitations.

Scholars have combined dimensional analysis with the experimental data and proposed some semi-empirical and semi-theoretical formulas to calculate the bubble velocity. In the inclined upward section of the water transmission pipeline, the air could be easily taken away due to buoyancy. In the horizontal pipe, Benjamin and Bakopoulos proposed a dimensionless number of 0.54 as the 
minimum flow rate for the bubbles starting to move; while Corcos suggested a dimensionless number of 0.484 for small diameter tubes [5]; and according to Escarameia's experiment [6], the critical velocity for bubble motion in the horizontal pipe was about $0.8 \mathrm{~m} / \mathrm{s}$, which was multiplied by a safety factor of 1.1. Therefore, the air could not be easily taken away when the water velocity is too small.

Actually, the air is most likely to accumulate in the inclined downward pipe because the buoyancy and the movement are in the opposite direction. As a result, study on bubble motion in the inclined downward pipe is much more than that in the inclined upward pipe or horizontal pipe, which has some reference significance for the bubble motion in the horizontal pipe. In the inclined downward pipe, it was considered that the air could be taken away when the water velocity was greater than the critical velocity $v_{c}$ which could be expressed as a dimensionless Froude number of $v_{c} /(g D)^{1 / 2}[7,8]$. Further on, the critical velocity was thought to be a function of Froude number, Reynolds number, pipe angle and surface tension [9]. Pozos [5] proposed a formula for the critical velocity through experiments taking the bubble size into account. Liu [10] thought the critical velocity for a single bubble was regardless of the bubble size in large-diameter pipes and proposed a minimum Froude number instead of the critical Froude number in rough pipes. Pothof and Clemens [11] proposed two formulas as the criteria for the flow regime transition in the inclined downward pipe. The air removal in the inclined downward pipe mainly rely on the water flow rate or the hydraulic jump at the bottom of an air pocket, and researchers have proposed different formulas for the calculation for the air entrainment by hydraulic jump under different conditions $[12,13,14,15]$.

Since too much study has been done on the bubble motion in the inclined downward pipe, and most parts of the water transmission pipeline appear to be horizontal or near horizontal, then study on bubble motion in the horizontal pipe could be equally important for the air removal. This article takes all the forces on the bubble into consideration and emphatically discusses the bubble velocity under bubble flow scenario in the horizontal pipe.

\section{Test rig and Methods}

Test Rig. The diagram of the test rig can be seen in Fig. 1 . The test rig is a circulating water supply system. The total length of the pipeline in the system is approximately $50 \mathrm{~m}$, and the inner and outer diameters of the pipe are $0.09 \mathrm{~m}$ and $0.11 \mathrm{~m}$ respectively. Most part of the pipeline is $0.6 \mathrm{~m}$ high above the ground except three special parts: the upward inclined part (length: $1.4 \mathrm{~m}$, angle: $45^{\circ}$ ), the horizontal part (length: $8 \mathrm{~m}$, height: $1.6 \mathrm{~m}$ ), and the downward inclined part (length: $1.4 \mathrm{~m}$, angle: $-45^{\circ}$ ). The pipeline is made of transparent plexiglass for easy observation. The flow is controlled by an electric control valve and measured by an ultrasonic flowmeter. The air is injected into the forepart of the pipeline by an air compressor and measured by a gas rotameter.

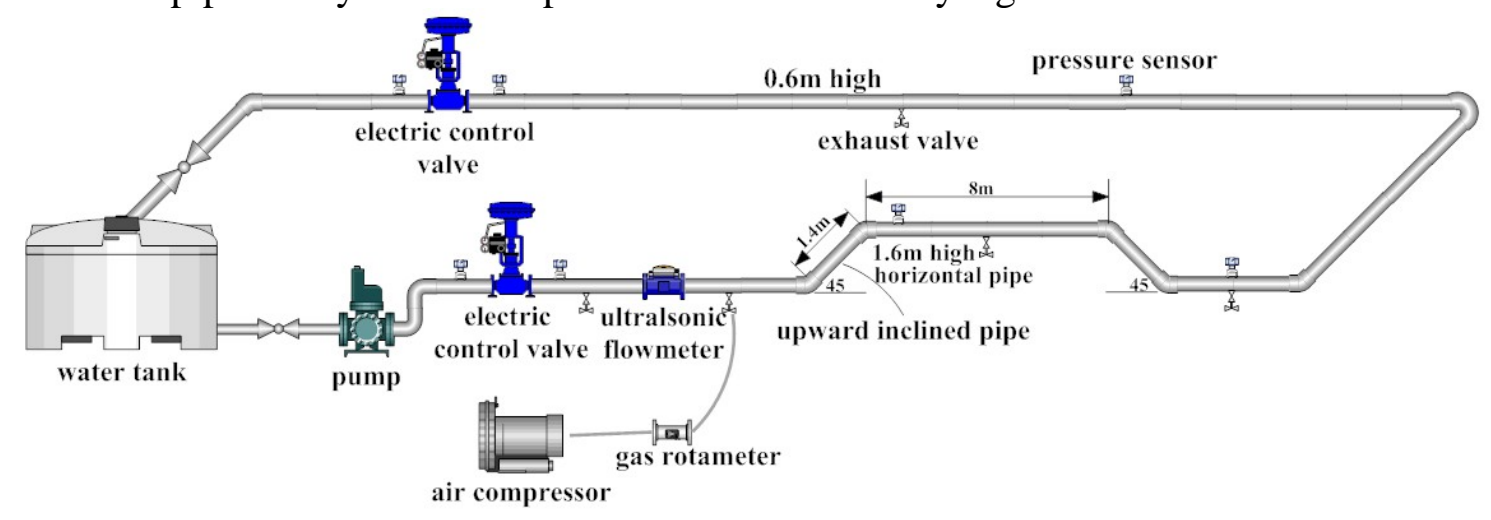

\section{Fig. 1 diagram of test rig}

Methods. In the experiment, an industrial camera (SONY XCD-SX910) was used to record images continuously from the high angle and the side angle of the pipe to capture the flow regimes of air-water two-phase flow and the motion of the bubbles, and the frame rate was set at $15 \mathrm{fps}$. In order to avoid the spray light, the camera was placed in an enclosed area with a light-proof cloth covering 
over a shelf. The illuminant for photographing was a rectangular light box (length: $1.2 \mathrm{~m}$, width: 0.2 $\mathrm{m}$ ) which can emit homogeneous white light, and it was always placed opposite to the camera lens. The distance between the pipe and the camera lens was always maintained at $0.7 \mathrm{~m}$ for the best photo effects. Firstly, the camera was placed at the side of the upward inclined pipe to capture the bubble motion in the static water. Secondly, the camera was used to capture the images of flow regimes and bubble motion in the horizontal pipe from the high angle, and the position for shooting is located at 5 $\mathrm{m}$ away from the upstream pipe elbow in order to make the air-water flow develop completely. 18 different water flow rates and 8 different air flow rates were picked out as the experimental operating conditions. The water flow rate ranged from 0 to $3(\mathrm{~m} / \mathrm{s})$ and the air flow rate were discrete values which are $0.5,1.0,1.5,2.0,2.5,3.0,3.5$ and $4.0\left(\mathrm{~m}^{3} / \mathrm{h}\right)$. Finally, the images taken in the experiment were used to analyze the bubble size and bubble velocity by using the image processing software (LabVIEW Vision Assistant, a scale ruler was placed beside the pipe which was helpful to identify the bubble size and the displacements of the bubbles), at the same time, the flow regimes in the horizontal pipe could be clearly observed.

\section{Results and Discussion}

Model Establishment. It is assumed that the bubble is a rigid body and would not change its shape during the motion process, so the friction between bubble and pipe wall can be calculated by the sliding friction formula. Viscous forces and surface tension do not play an important part in the water transmission pipe so that can be neglected. The diagram of force analysis of a bubble in the inclined upward pipe is shown in the Fig. 2.
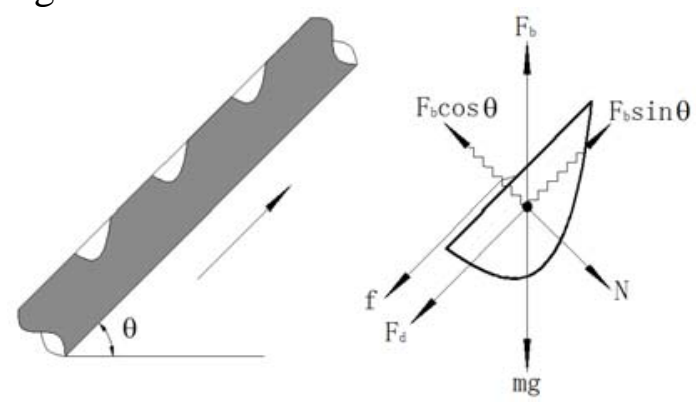

Fig. 2 diagram of force analysis of a bubble in inclined upward pipe

When the bubble is in a steady motion state, it is in the equilibrium condition of forces which includes gravity $(m g)$, buoyancy $\left(F_{b}\right)$, drag force $\left(F_{d}\right)$, supportive of wall $(N)$, and friction of wall $(f)$. According to Newton's second law of motion, it can be obtained:

$F_{b} \sin \theta-F_{d}-m g \sin \theta-f=0$.

(i) $F_{b}=\rho_{w} g V_{b}, \rho_{w}$ is the water density, $10^{3} \mathrm{~kg} / \mathrm{m}^{3} ; g$ is the acceleration of gravity, $9.8 \mathrm{~m} / \mathrm{s}^{2} ; V_{b}$ is the volume of the bubble.

(ii) $F_{d}=C_{d} \rho_{w} \frac{\left(v_{b}-v_{w}\right)^{2}}{2} A_{b}, C_{d}$ is the drag force coefficient that is related to Reynolds number.

The Reynolds number calculated from the slip velocity between the bubble and the water generally ranges from $10^{3}$ to $10^{5}$ in the water transmission pipe. Assuming the bubble is a sphere, $C_{d}$ can be regarded as a constant of 0.45 ; $v_{w}$ is the water velocity; $v_{b}$ is the bubble velocity; $A_{b}$ is the projected area of the bubble that is perpendicular to the flow direction.

(iii) $m g \sin \theta \approx 0$, which is due to the small density of air, so that the gravity of the bubble can be ignored.

(iv) $f=\mu N=\mu F_{b} \cos \theta, N=F_{b} \cos \theta-m g \cos \theta \approx F_{b} \cos \theta, \mu$ is the sliding friction coefficient between the bubble and the pipe wall; $\theta$ refers to the inclined angle between the pipe and the 
horizontal direction, and when the water flows in the inclined upward pipe, $\theta>0$, while the water flows in the inclined downward pipe, $\theta<0$.

Thus, Eq. 1 can be reduced to:

$$
v_{b}=v_{w} \pm \sqrt{\frac{2 g V_{b}|\sin \theta-\mu \cos \theta|}{C_{d} A_{b}}} .
$$

Eq. 2 is a general expression of the bubble velocity for all angles from $0^{\circ}$ to $90^{\circ}$, and it is concluded that the bubble velocity in the inclined upward pipe is related to the inclined angle and the sliding friction coefficient between the bubble and the pipe wall.

Hesketh et al. suggested an expression to describe the mean diameter of the bubbles under bubble flow as follow [16]:

$$
d_{b}=0.8556\left(W e_{c r i t}^{\prime}\right)^{0.6}\left(\frac{\sigma_{w}^{0.6}}{\rho_{w}^{0.3} \rho_{g}^{0.2} \mu_{w}^{0.1}}\right)\left(\frac{D^{0.5}}{v_{w}^{1.1}}\right),
$$

$d_{b}$ is the mean diameter of the bubbles; $W e^{\prime}{ }_{c r i t}$ is the critical Weber number of 1.1 which is obtained through experiments; $\sigma_{w}$ is the surface tension of water which takes a constant value of $72.8 \times 10^{-3}$ $\mathrm{N} / \mathrm{m}$ when the temperature is $20{ }^{\circ} \mathrm{C}$; $\rho_{g}$ is the density of air and can be expressed as Eq. 4 ; $\mu_{w}$ is the coefficient of dynamic viscosity of water that is related to the temperature, which is expressed in Eq. 5.

$$
\begin{aligned}
& \rho_{g}=1.293 \times\left(\frac{H+10.34}{10.34}\right)\left(\frac{273.15}{T+273.15}\right), \\
& \mu_{w}=\left(1.79155-0.06119 T+0.00148 T^{2}-1.88889 \times 10^{-5} T^{3}\right) \times 10^{-3}(0 \leq T \leq 30) .
\end{aligned}
$$

$H$ is the pipe pressure (m); $T$ is the temperature of water $\left({ }^{\circ} \mathrm{C}\right)$. Supposing the bubble is a sphere with a diameter of $d_{b}$ and $\theta=0^{\circ}$, Eq. 2 can be simplified as:

$$
v_{b}=v_{w}-\sqrt{29.037 \mu d_{b}} \text {. }
$$

From the above, the bubble velocity in the horizontal water transmission pipe under bubble flow condition can be calculated through Eq. 2, Eq. 3, Eq. 4 and Eq. 5.

Model Verification. The sliding friction coefficient between the bubble and the pipe wall can be obtained through the experiment of bubble motion in the inclined upward pipe. The diagram of bubble sizes can be seen in Fig. 3. The statistical data of bubble sizes and the bubble velocities in the experiment are shown Table 1 and Fig. 4.

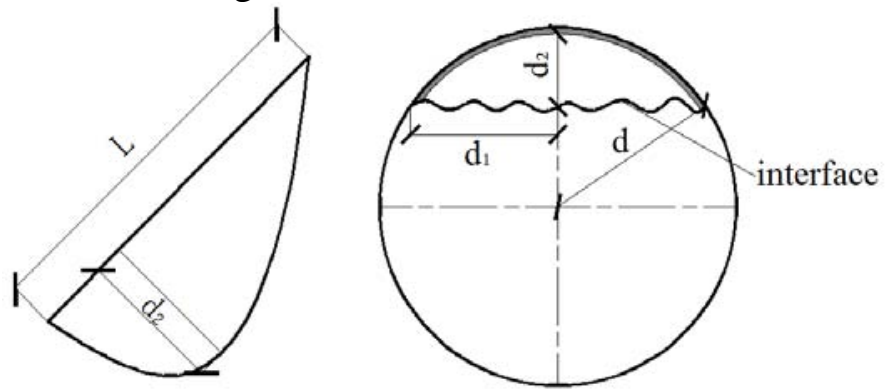

Fig. 3 Diagram of bubble size in inclined upward pipe 
Table 1 Statistical data of bubble sizes

\begin{tabular}{cc|ccccc}
\hline NO. & size & $L[\mathrm{~mm}]$ & $d_{2}[\mathrm{~mm}]$ & $d_{1}[\mathrm{~mm}]$ & $A_{b}\left[\mathrm{~cm}^{2}\right]$ & $V_{b}\left[\mathrm{~cm}^{3}\right]$ \\
\hline 1 & 53.10 & 29.14 & 42.11 & 17.84 & 31.57 \\
2 & 52.67 & 26.75 & 41.13 & 15.85 & 27.82 \\
3 & 43.57 & 20.25 & 37.58 & 10.71 & 15.56 \\
4 & 58.52 & 27.4 & 41.42 & 16.38 & 31.96 \\
\hline
\end{tabular}
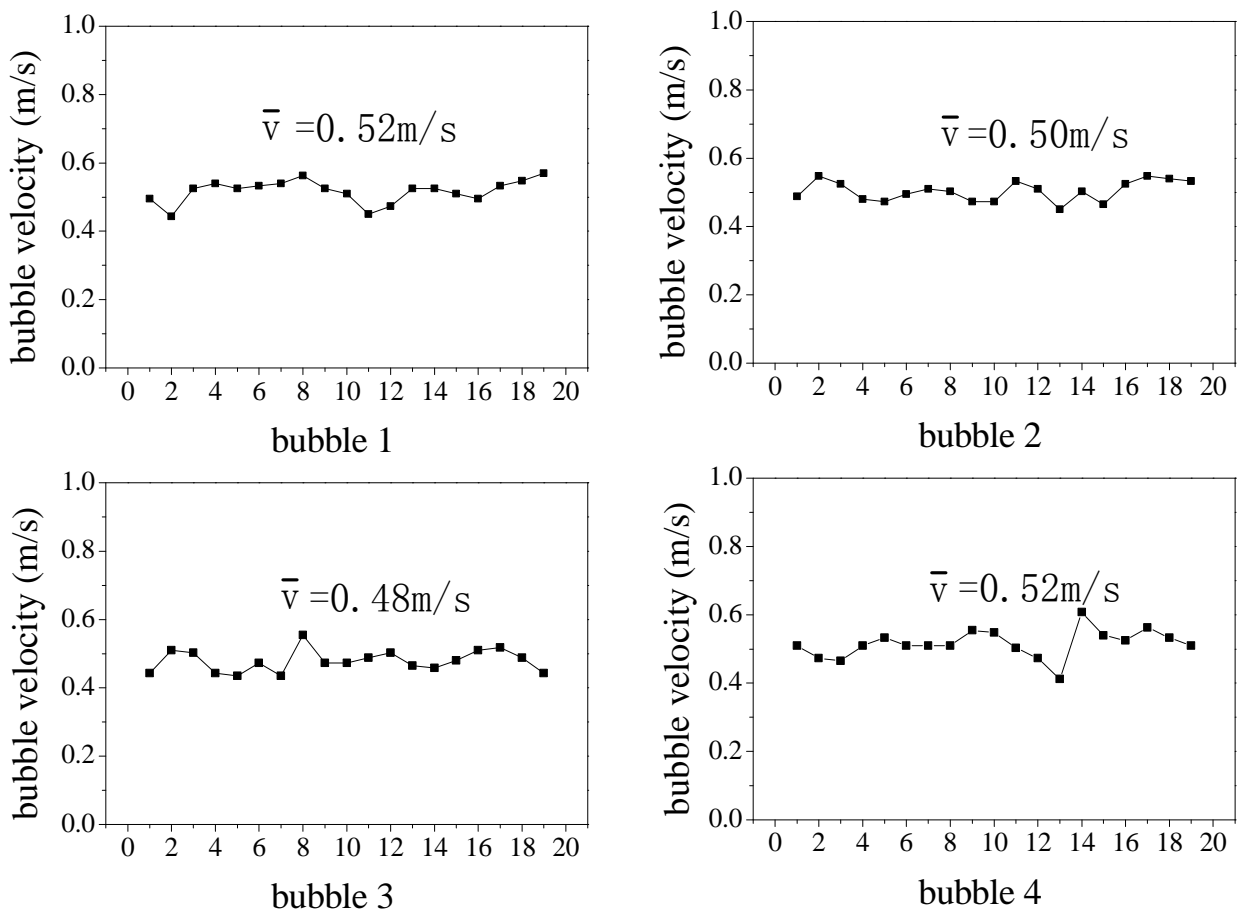

Fig. 4 Experimental data of bubble velocity in inclined upward pipe

The mean value of $\mu$ can be calculated by taking the experimental data in the inclined upward pipe into Eq. 2, and the mean value of $\mu$ equals to 0.53 . The experimental data in the horizontal pipe is listed in Table 2, and if taking the experimental data and calculated $\mu$ into Eq. 2, Eq. 3, Eq. 4 and Eq. 5 , it can theoretically obtain the bubble velocities and bubble diameters which are also listed in Table 2. The comparison of theoretical data and experimental data is shown in Fig. 5.

Table 2 Experimental and theoretical value of bubble size in bubble flow scenario

\begin{tabular}{cccccccccc}
\hline NO. & 1 & 2 & 3 & 4 & 5 & 6 & 7 & 8 & 9 \\
\hline water velocity [m/s] & 1.81 & 1.93 & 2.06 & 2.18 & 2.32 & 2.42 & 2.58 & 2.84 & 2.96 \\
pipe pressure [m] & 2.77 & 3.18 & 3.60 & 3.99 & 4.42 & 4.79 & 5.38 & 6.45 & 6.87 \\
experimental data [mm] & 6.0 & 5.6 & 5.2 & 5.0 & 4.6 & 4.4 & 4.1 & 3.7 & 3.5 \\
Theoretical data [mm] & 6.59 & 6.11 & 5.64 & 5.27 & 4.90 & 4.66 & 4.31 & 3.83 & 3.63 \\
\hline
\end{tabular}

As Fig. 5a shows, the experimental values for mean bubble diameter are slightly smaller than the theoretical values. However, the error between them has little effect on the calculation of bubble velocities as shown in Fig. 5b. During the experiment, when the water velocity was greater than 2.5 $\mathrm{m} / \mathrm{s}$, it was difficult to identify the bubble velocity by image analysis due to the unstable bubble clusters, so that available experimental data is less than theoretical data as Fig. $5 b$ shows. It is also shown in Fig. 5b that the experimental values are proportional to the theoretical values. Therefore, in order to obtain a more accurate result, Eq. 6 is corrected by multiplying a correcting factor of 0.93 , and then Eq. 6 becomes:

$$
v_{b}=0.93 \times\left(v_{w}-\sqrt{29.037 \mu d_{b}}\right) .
$$



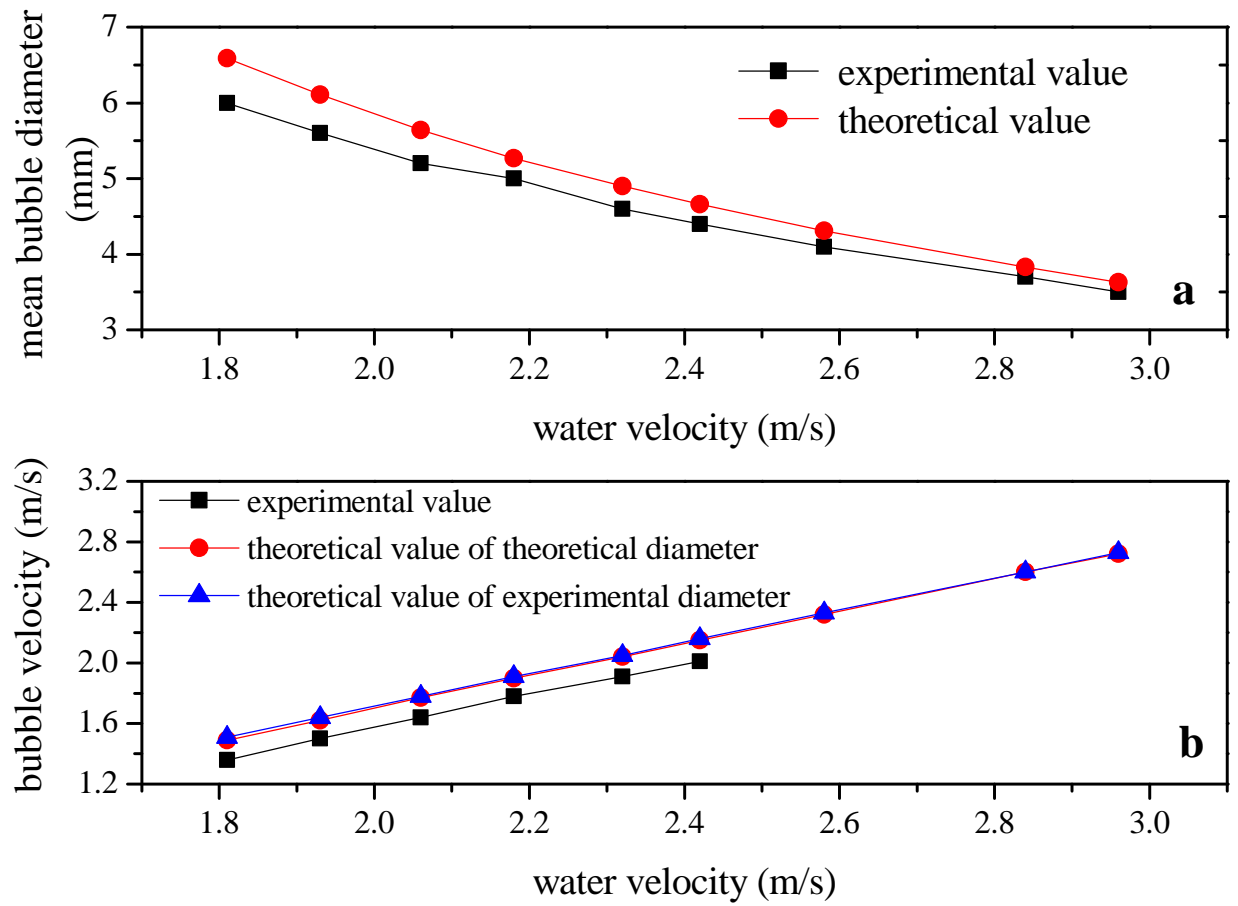

Fig.5 Comparison of experimental and theoretical data on mean bubble diameter and bubble velocity under bubble flow scenario

Model Application. The effects of pipe diameter, pipe pressure, pipe material and temperature on the bubble diameter and the bubble velocity were discussed and analyzed, and the results are shown in Fig. 6. The sliding friction coefficient $\mu$ was obtained through experiments as mentioned above, but it is unrealistic to obtain the coefficients for all the other pipe materials. It is known that $\mu$ is positively correlated to the roughness of the pipe wall, thus, assuming $\mu$ is proportional to the roughness coefficient of the pipes, then the sliding friction coefficients of different pipes can be calculated in Table 3 on the basis of the plexiglass pipe's $\mu$.
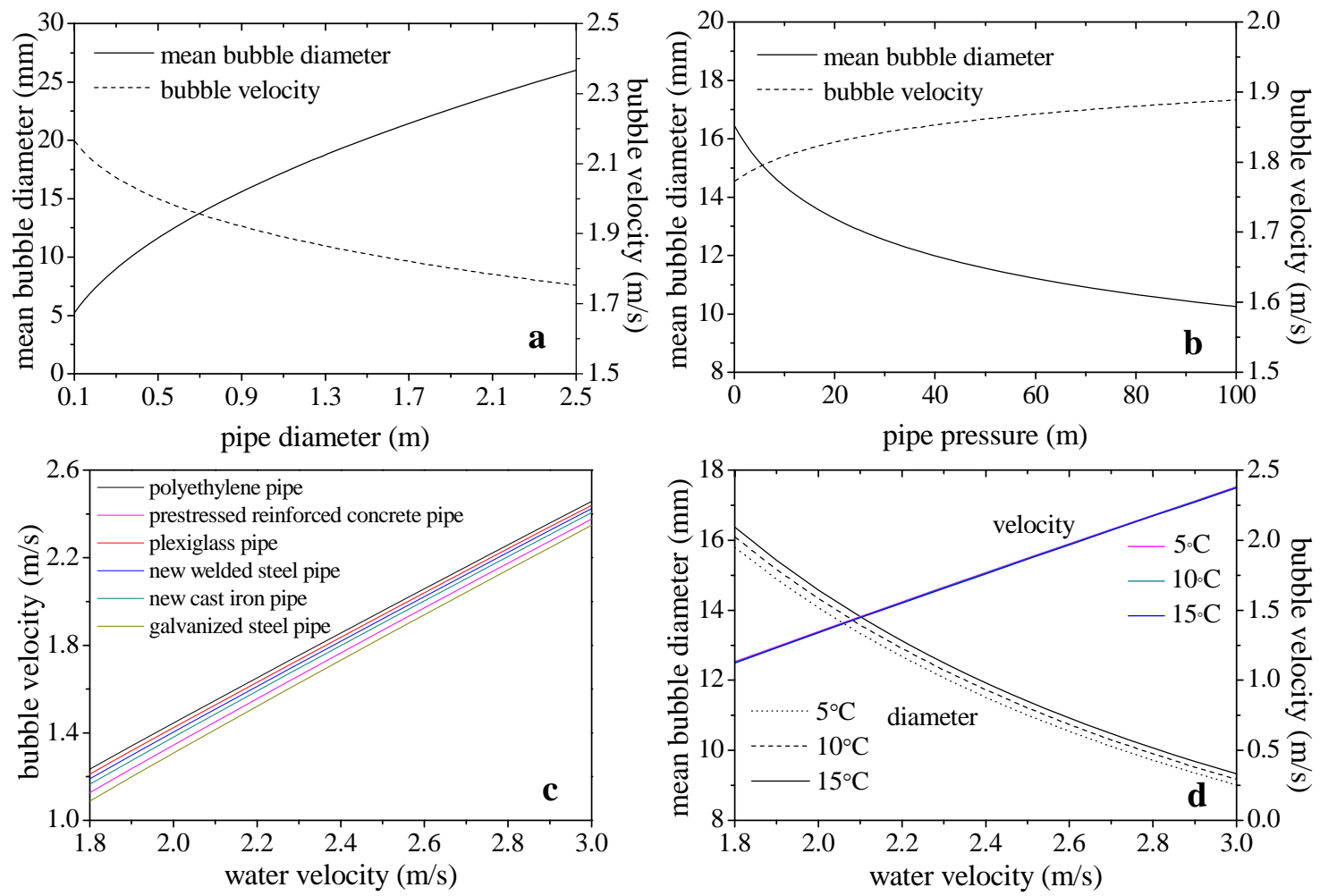

Fig. 6 Effects of pipe diameter, pipe pressure, pipe material and temperature on the bubble diameter and the bubble velocity 
Table 3 sliding friction coefficients of different pipe materials

\begin{tabular}{ccc}
\hline pipe materials & roughness coefficient & Sliding friction coefficient \\
\hline plexiglass pipe & 0.01 & 0.53 \\
polyethylene pipe & 0.009 & 0.477 \\
prestressed reinforced concrete pipe & 0.014 & 0.742 \\
galvanized steel pipe & 0.016 & 0.848 \\
new cast iron pipe & 0.012 & 0.636 \\
new welded steel pipe & 0.011 & 0.583 \\
\hline
\end{tabular}

In Fig. 6a, under the flow conditions of $v_{w}$ is $2.5 \mathrm{~m} / \mathrm{s}, H$ is $0 \mathrm{~m}$, and $T$ is $10^{\circ} \mathrm{C}$, the mean bubble diameter increases and the bubble velocity decreases as the pipe diameter increases. The air is more difficult to be taken away in the pipe with large diameter. In Fig. 6b, under the flow conditions of $v_{w}$ is $2.5 \mathrm{~m} / \mathrm{s}, D$ is $1 \mathrm{~m}$, and $T$ is $10^{\circ} \mathrm{C}$, the mean bubble diameter decreases and the bubble velocity increases as the pipe pressure increases. The bubbles in the area with low pressure cannot be easily taken away. In Fig. 6c, under the flow conditions of $D$ is $1 \mathrm{~m}, H$ is $0 \mathrm{~m}$, and $T$ is $10^{\circ} \mathrm{C}$, the bubble moves more slowly in the rougher pipe. In Fig. 6d, under the flow conditions of $D$ is $1 \mathrm{~m}, H$ is $0 \mathrm{~m}$, and $\mu$ is 0.74 , the mean bubble diameter increases as the water temperature rises, and temperature has little effect on the bubble velocity.

\section{Conclusion}

In this paper, a model for calculating bubble velocity in the horizontal pipe under bubble flow scenario is established on the basis of theoretical stress analysis. Although the calculated results of the model have a little difference with the experimental data, and after correction it is completely feasible to calculate the bubble velocity in the actual engineering. The model tells that the air in the pipes with large diameter or coarse inner surface is relatively difficult to be taken away by water. The model also shows that the greater the bubble diameter is and the slower the bubble moves. In addition, it is suggested that more air release valves should be installed at the terminal part of the pipeline where is in a low pressure area because the air moves more slowly in the low pressure areas.

\section{Acknowledgements}

This work was financially supported by National Natural Science Foundation of China (51178141) and National Major Project: Water Pollution Control and Management Technology (2012ZX07408-002-004-002).

\section{References}

[1] Lei Liu, Fangde Zhou: Measurement Technique Vol. 4 (1996), p. 17-18. (In Chinese)

[2] Feng Dong, Xutong Qiao, Zhixu Jiang, et al: Journal of Tianjin University Vol. 37-6 (2004), p. 510-514. (In Chinese)

[3] Jiansheng Zhang, Qing Lv, Chuandong Sun, et al: ACTA PHOTONICA SIN ICA Vol. 29-10 (2000), p. 952-955.

[4] Wei Luo, Xiaode Zhou, Wen Cheng, et al: Transducer and Microsystem Technologies Vol. 25-2 (2006), p. 1-3, 6. (In Chinese)

[5] O. Pozos, C.A. Gonzalez, J. Giesecke, et al: Journal of hydraulic research Vol. 48-3 (2010), p. 338-347.

[6] M. Escarameia: Water Manag. ICE Vol. 160-WM1 (2007), p. 25-34.

[7] A.A. Kalinske, P.H. Bliss: Civil Eng Vol. 13-10 (1943), p. 480-482.

[8] C.S. Martin: J. Fluids Eng. ASME Vol. 98-4 (1976), p. 715-722. 
[9] K.H. Bendiksen: Intl. J. Multiph. Flow Vol. 10-4 (1984), p. 467-483.

[10] Ting Liu, Y. James: Journal of hydraulic research Vol. 51-3 (2013), p. 265-272.

[11] P. Ivo, C. Francois: Journal of hydraulic research Vol. 48-4 (2010), p. 499-503.

[12] D.A. Ervine: Proc. Instn Civ Engrs Wat., Marit.\& Energy, Sept-130 (1998), p. 142-153.

[13] P. Ivo, C. Francois: International Journal of Multiphase Flow Vol. 37 (2011), p. 278-292.

[14] D.M. Joshua, L.B. Steven, C.J. Michael: Journal of hydraulic research Vol. 49-1 (2011), p. 90-95.

[15] P. Ivo, C. Francois: 9th International Conference on Urban Drainage Modelling, Belgrade (2012), p. 1-11.

[16] R.P. Hesketh, T.W. Fraser Russell, A.W. Etchells. Bubble size in horizontal pipelines. AIChE journal, Vol. 33-4 (1987), p. 663-667. 